Memorias del VII Encuentro Nacional de Experiencias en la Enseñanza de la Biología y la Educación Ambiental y II Congreso Nacional de Investigación en la Enseñanza de la Biología

\title{
EL CONOCIMIENTO DEL CONTEXTO COMO UN CONOCIMIENTO PROFESIONAL DEL PROFESOR DE BIOLOGÍA. APORTES DE CUATRO DOCENTES Y SUS EXPERIENCIAS.
}

\section{THE KNOWLEDGE OF CONTEXT AS A KNOWLEDGE PROFESSIONAL OF PROFESSOR OF BIOLOGY. CONTRIBUTIONS OF FOUR TEACHERS AND THEIR EXPERIENCES.}

\section{David Osorio Vanegas ${ }^{1}$}

Yesika Hernández González ${ }^{2}$

Raquel Soto Soto ${ }^{3}$

Yónier Orozco Marín ${ }^{4}$

\section{Resumen}

Las categorías del conocimiento profesional del profesor fueron planteadas por Shulman en 1986 y 1987, a pesar de que una de ellas corresponde al conocimiento del contexto educativo, se hace necesario profundizar sobre su significado y relación con otros conocimientos del profesor, particularmente para el profesor de biología. Se presentan a través de un marco interpretativo los aportes de tres profesores en formación y un docente con cerca de cinco años de experiencia en la enseñanza de la biología referentes al conocimiento del contexto al plantear de manera individual una definición de contexto, la relación del contexto con la enseñanza de la biología y relatar algunas actividades y experiencias puestas en práctica por cada uno de los docentes donde se hubiera intencionado la vinculación del contexto al proceso de enseñanza aprendizaje a algún concepto biológico. A partir de la sistematización de estos resultados se encuentra que el conocimiento del contexto se constituye desde diferentes significados y perspectivas para cada docente, su relación con la enseñanza y aprendizaje de la biología es bidireccional, y se encuentra vinculado principalmente al conocimiento de las orientaciones, estrategias y de los estudiantes.

Palabras Clave: Conocimiento del contexto, Conocimiento profesional del docente de biología, orientaciones y estrategias.

\section{Abstract}

The categories of teachers' professional knowledge were raised by Shulman in 1986 and 1987, even though one of them corresponds to the knowledge of the educational context, it is necessary to elaborate on its meaning and relationship to other teacher's knowledge, particularly for teacher biology. Is presented

\footnotetext{
${ }^{1}$ renhyus@hotmail.com

2 jesica mayuli90@hotmail.com

3 angel09@hotmail.com

4 apmusicomano@gmail.com
} 
Memorias del VII Encuentro Nacional de Experiencias en la Enseñanza de la Biología y la Educación Ambiental y II Congreso Nacional de Investigación en la Enseñanza de la Biología

through an interpretive framework the contributions of three teachers in training and teachers with about five years of experience in the teaching of biology concerning the knowledge of the context to individually pose a definition of context, the relationship context with the teaching of biology and relate some activities and experiences implemented by each of the teachers where they had intended context linking the teaching-learning process to a biological concept. Since the systematization of these results is that knowledge of context From various meanings and perspectives for each teacher, his relationship with the teaching and learning of biology is bidirectional, and is primarily linked to the knowledge of the guidelines, strategies and students.

Keywords: Knowledge of context, teachers' professional knowledge of biology, guidelines and strategies.

\section{Introducción.}

Anteriormente la mayoría de estudios encontraban como los estudiantes aprendían, sus dificultades y las preconcepciones o ideas previas que estos poseían, también se hallaban nociones de que la apropiación del docente hacia sus clases podía influir en ello,pero eran muy pocos los estudiosrealizadosque daban cuenta de este aspecto. Sin embargo desde 1986 se elaboraron los primeros estudios sobre el conocimiento profesional del profesor donde Shulman, identifico tres categorías del conocimiento profesional del profesor: conocimiento disciplinar, el conocimiento didáctico del contenido (CDC ó PCK), y conocimiento curricular; luego en 1987 establece siete categorías en total, elconocimiento del contenido,el conocimiento didáctico,donde hace referencia a las estrategias de manejo y organización de la clase, conocimiento del currículo, como todos los programas que sirven de herramientas para el oficio del docente, conocimiento didáctico del contenido (CDC ó PCK), el cual lo define como una amalgama entre la materia y la pedagogía, el conocimiento de los alumnos,el conocimiento de los contextos educativos, y finalmente el conocimiento de los objetivos, finalidades y valores educativos.

El conocimiento del contenido didáctico (CDC ó PCK), es definido como "La capacidad del maestro de transformar el conocimiento del contenido que éste posee en formas que son poderosas pedagógicamente hablando, y además que se adapten a las variaciones en la habilidad y el trasfondo presentado por los estudiantes",(Shulman, 1987, p 15). Más tarde, autores como Magnusson en 1989, por medio de otros estudios profundizan en la categoría del conocimiento didáctico del contenido (CDC ó PCK), explicando que el PCK comprende 5 componentes; 1.Orientaciones sobre la enseñanza de las ciencias, 2. Conocimiento acerca de las ideas de los estudiantes, 3. Conocimiento de las estrategias de enseñanza, 4. Conocimiento del currículo y 5. Conocimiento de la evaluación. 
Edición Extra-Ordinaria. ISSN 2027-1034 P.p $867-876$

Memorias del VII Encuentro Nacional de Experiencias en la Enseñanza de la Biología y la Educación Ambiental y II Congreso Nacional de Investigación en la Enseñanza de la Biología

En otros estudios recientes, Valbuena (2007) Identifica las características que debe tener un profesor de biología y del conocimiento que éste requiere para enseñar contenidos específicos de biología, afirmando que el CDCB (conocimiento del contenido didáctico de la biología) se produce a partir del proceso de transformación, procesamiento e interrelación de múltiples componentes como lo son: los Contenidos, las finalidades y la enseñanza de la biología, las estrategias metodológicas, las concepciones de los alumnos, las dificultades en el proceso enseñanza-aprendizaje de la biología, y la evaluación de los aprendizajes.

Sobre la categoría del conocimiento sobre el contexto, Fernández, Balboa y Stiehl (198) citados por Kind (2009)expresan que la inclusión del mismo, era algo nuevo pues notaron que las barreras contextuales contribuyen a la práctica propia de la docencia universitaria incluyendo el manejo de grupos grandes, límites de tiempo, escasez de recursos apropiados, actitudes de los estudiantes y problemáticas de permanencia y promoción, para lo cual el contexto influía en el CDC, dicho de otra manera "el conocimiento cotidiano está frecuentemente influenciado por la relación del sujeto con los elementos contextuales a diferentes niveles (sociedad, cultura, familia, escuela, etc)." (Valbuena 2007 p 71), y que en la medida en la que se reconocen estos aspectos sociales, motivacionales, las capacidades metacognitivas y por ende los procesos cognitivos del estudiante, el docente enriquece su CDCB y además lo usa para desarrollar contenidos y actividades de enseñanza acordes a estos elementos. Desde otros puntos de vista, otros autores como Carlsen (1999) citado por Valbuena, generan relaciones entre el conocimiento del contexto con las demás categorías.Morine, Dershimer y Kent (1999) citados también por Valbuena aseveran que esta categoría posee influencia sobre el conocimiento pedagógico (referente a la organización y administración de la clase). Y Rodrigo (1997) afirma que el contexto sociocultural posee gran influencia en el conocimiento de los estudiantes ya que organizan las teorías implícitas de los mismos sobre diferentes conceptos en tendencias ó las determina en diferentes grupos sociales.

No obstante, Bermúdez y Longhi (2012), realizan otras precisiones sobre el conocimiento del contexto afirmando que en las clases se encuentran presentes tres tipos de contextos: el situacional que se refiere al medio sociocultural, ambiental, institucional y al momento histórico; el contexto lingüístico representado en el habla de profesores y alumnos, y en la terminología propia del contenido y su lógica; y el contexto mental del docente y alumnos, conformado por todos lo "no observable" mencionados -como las representaciones y referentes sobre el tema (De Longhi, 2000). Esta autora afirma que el conocimiento sobre estos contextos permiten moldear el conocimiento del contenido didáctico y de la materia, ya que adaptan los contenidos, las estrategias, las evaluaciones y el momento del año en que deben ocurrir estas intervenciones, a su vez esta confluencia entre conocimientos permite que se tenga en cuenta el entorno en el que está inmersa la institución y las problemáticas presentes por medio de las cuales la 
Memorias del VII Encuentro Nacional de Experiencias en la Enseñanza de la Biología y la Educación Ambiental y II Congreso Nacional de Investigación en la Enseñanza de la Biología

clase se convierte en el medio para hacer un reconocimiento más profundo de estas problemáticas, generar proyectos de investigación, incorporar el conocimiento tradicional de los estudiantes sobre su medio y para promover el uso del lenguaje apropiado por medio de la argumentación, generación de hipótesis, interpretación y discusión de los resultados encontrados.

De acuerdo a lo anterior, podemos decir que sobre el conocimiento profesional del profesor, diversos autores se han encargado de profundizar en la categoría del conocimiento didáctico del contenido, pero la categoría del conocimiento del contexto ha sido analizada muy tácitamente por Shulman (1987) indicando que va desde el funcionamiento del grupo, la gestión y el carácter de las comunidades y culturas, y de acuerdo a revisiones realizadas se observa quemuy pocos son los autores que sostienen que este hace parte del conocimiento profesional del profesor, que posea influencias en el conocimiento del contenido didáctico, osu relación con la enseñanza de la biología, por lo tanto este trabajo tuvo como objetivo reconocer elementos del conocimiento del contexto como un conocimiento profesional del profesor de biología y sus posibles relaciones con otros conocimientos, a través del análisis y reflexión de la práctica docente de cuatro profesores de biología, tres de ellos en proceso de formación que se encuentran realizando sus prácticas pedagógicas (Caso 1, 2 y 3) y un profesor con cerca de cinco años de experiencia (Caso 4).

\section{Metodología}

Los resultados de la investigación a presentar se fundamentan en los planteamientos realizados por Arnal \& Latorre (1992) en relación al paradigma de investigación interpretativo hermenéutico definido como:

"El interés se centra en el estudio de los significados de las acciones humanas y de la vida social (...) Enfatiza la compresión e interpretación de la realidad educativa desde los significados de las personas implicadas en los contextos educativos y estudia sus creencias, intenciones, motivaciones y otras características del proceso educativo no observables directamente ni susceptibles de experimentación" (Arnal \& Latorre, 1992 p. 41).

Cada uno de los cuatro docentes a partir de su experiencia como docente de biología planteó su definición de contexto, la relación que el contexto presenta con la enseñanza de la biología acompañado de algunos ejemplos de su práctica sobre esta relación. A partir de estos elementos se resaltaron los elementos principales (comunes o no) de las perspectivas de los cuatro docentes referentes a la relación del contexto con los procesos de enseñanzaaprendizaje de la biología y del contexto como un conocimiento profesional del profesor de biología.

\section{Resultados Y Discusión}


Memorias del VII Encuentro Nacional de Experiencias en la Enseñanza de la Biología y la Educación Ambiental y II Congreso Nacional de Investigación en la Enseñanza de la Biología

\section{Definición de contexto}

Desde la perspectiva de los docentes participantes de esta investigación, el conocimiento del contexto se constituye desde diferentes significados y perspectivas, donde la propia experiencia, formación, historia de vida y personalidad intervienen en la construcción de representaciones sobre este término. Desde la clasificación que Bermúdez y Longhi (2012) proponen de los contextos presentes en el aula, la posición de estos docentes se encuentra relacionada con una perspectiva del contexto situacional, el contexto se entiende como el "conjunto de dinámicas socio-ecológicas que ocurren en el espacio donde profesor, estudiante y escuela se desenvuelven todos los días, así como a las situaciones e informaciones a las que constantemente se enfrentan (caso 1)" o las "características sociales, culturales, económicas, ecológicas, psicológicas y conceptuales en las que está inmerso el estudiante (Caso 4)", posiciones en las cuales es de destacar que además de los diferentes factores de este contexto, son de gran influencia las informaciones y situaciones que estos generan y en los cuales no solo estudiantes, sino también docentes y escuela se hacen participes, lo que sugiere que el profesor puede hacer énfasis de manera explícita en estas informaciones y situaciones en las que se ven inmersos en la escuela, e incorporarlas a los procesos de enseñanza-aprendizaje en las clases de biología. Para Barnett y Hodson (2001) La enseñanza es "contexto-específica" y, por tanto, las decisiones de los docentes en el aula están determinadas por el contexto, por las condiciones sociales y culturales específicas, lo anterior seguramente no es puesto en discusión por nadie, pero desde la posición de los docentes caso 2 y 3 , no es suficiente comprender el contexto desde una relación unidireccional en la que éste interfiere en los procesos de enseñanza-aprendizaje en este caso de la biología, sino que la relación puede ser bidireccional ya que el contexto se refiere al "entorno social, geográfico y ambiental, donde constantemente se establecen relaciones entre los sujetos, donde los estudiantes conocen aspectos del mismo y pueden establecer vínculos con demás miembros de su comunidad en pro de mejorarlo (caso 2)" o a "todas las interacciones que ocurren alrededor del sujeto, que lo pueden afectar pero que a su vez el sujeto también puede transformar (caso 3)", es decir, el contexto no debe ser concebido únicamente como uno de los componentes que permean los procesos de enseñanza-aprendizaje de la biología, sino que estos mismos procesos pueden transformar situaciones (por lo menos próximas) del contexto en los que tanto profesor y estudiantes se encuentran inmersos.

\section{Relación del contexto con la enseñanza de la biología - Ejemplos y experiencias}

Las relaciones que los docentes expresan que se presentan entre el conocimiento del contexto y la enseñanza de la biología se enmarcan en dos líneas principales: 
Edición Extra-Ordinaria. ISSN 2027-1034 P.p $867-876$

Memorias del VII Encuentro Nacional de Experiencias en la Enseñanza de la Biología y la Educación Ambiental y II Congreso Nacional de Investigación en la Enseñanza de la Biología

1. El conocimiento del contexto y su relación con las orientaciones de enseñanza y de aprendizaje de la biología:

A pesar de que el conocimiento sobre las orientaciones por parte del profesor, ha sido principalmente desarrollado como uno de los conocimientos del CDC, más que una categoría del conocimiento profesional del profesor, se hace evidente que para los profesores participantes en este trabajo, como lo manifiesta el caso 3, el contexto se relaciona como un "elemento que moviliza el proceso de enseñanza de la biología debido a que permite generar las orientaciones u objetivos de la clase" que de hecho pueden ser vinculadas a que "los estudiantes enriquezcan o amplíen su visión sobre el mismo contexto" donde el objetivo de aprendizaje "no se puede desligar de conocer el contexto donde viven los estudiantes (caso 2)", ó "el contexto como fundamento y presupuesto para la definición de propósitos y objetivos (caso 1)" en el abordaje de cualquier concepto en la clase de biología. Desde esta perspectiva del contexto como referente para la planeación y definición de objetivos de aprendizaje se trasciende la corriente de reconocer la estructura del conocimiento biológico, sus procedimientos y conceptos estructurantes (Castro y Valbuena, 2007), sino que además se hace necesario reconocer que pensar en organizar los procesos de enseñanza aprendizaje de estos conceptos estructurantes, si bien, contribuye a mejores comprensiones sobre lo vivo por parte de los estudiantes, estas pueden ser más significativas cuando estos conceptos más que presentar una coherencia entre ellos mismos, a la vez presentan coherencia y relación con verdaderas problemáticas del contexto situacional donde estudiante, profesor, escuela y comunidad están inmersos, con el fin de que los estudiantes se aproximen a la comprensión de las interacciones, problemáticas y posibles vías de transformación de su contexto y a la vez desarrollen una comprensión no solo más estructurada y organizada (desde el punto de vista biológico) de los fenómenos relacionados con lo vivo, sino también más situada. El caso 4 lo hace más explicito cuando asegura que "es prioridad conocer el contexto de los estudiantes como punto de partida para el diseño curricular, el contexto a tratar se puede conocer con los siguientes ítems: socio-cultural, económico, ecológico, psicológico y conceptual", donde a partir del conocimiento de estos aspectos del contexto situacional se pueden seleccionar no solo orientaciones para el aprendizaje, también contenidos, estrategias, formas de evaluación, entre otros. Cañal (2004) propone como objetivos prioritarios para la enseñanza de la biología aquellos que se refieran al desarrollo de capacidades e instrumentos cognitivos y afectivos necesarios para que los estudiantes logren comprender, posicionarse personalmente y en su caso actuar ante problemas de corte socio-ambiental que incluyan componentes del conocimiento biológico, lo cual representa un soporte a la hipótesis construida por los docentes participantes de este trabajo.

2. Las estrategias de enseñanza y de aprendizaje de la biología en vinculación al contexto. 
Edición Extra-Ordinaria. ISSN 2027-1034 P.p 867 - 876

Memorias del VII Encuentro Nacional de Experiencias en la Enseñanza de la Biología y la Educación Ambiental y II Congreso Nacional de Investigación en la Enseñanza de la Biología

Las estrategias que los docentes vinculados a este trabajo manifiestan que han implementado y en las cuales se hace relación intencionada del contexto a estas estrategias, se pueden entender en tres vías: Estrategias adaptadas a las características de los estudiantes (por parte del profesor), estrategias de reconocimiento y aprendizaje del contexto, y estrategias para la transformación y acción sobre el contexto (estas dos últimas desarrolladas por los estudiantes).

Bajo la primera vía, en particular el caso 4 (docente con experiencia), considera que teniendo en cuenta las características económicas, sicológicas, sociales entre otras de los estudiantes, estas deben determinar el tipo de actividades concretas que el profesor plantea en el aula, e incluso las posiciones que debe tomar ante ciertas situaciones, al entender estos comportamientos y reflexiones como el producto de la influencia de las interacciones e informaciones del contexto sobre sus estudiantes, en este sentido el docente plantea que "no solo podemos hablar sobre la biodiversidad del Amazonas o el ecosistema desértico, cuando los estudiantes no conocen bien su barrio y lo consideran que no es parte de la naturaleza" o cuando el "docente reclama a una joven por su falta de atención en la clase, que se encuentra echando globos en el salón, cuando en realidad la estudiante está preocupada porque no sabe como decirle a sus padres que está embarazada", para este docente "la motivación y el aspecto psicológico son necesarios para el desarrollo con éxito de una clase", en términos de Marcelo (1999) el profesor necesita conocer las características socio-económicas y culturales del barrio, las expectativas de sus estudiantes, entre otros aspectos, los cuales pueden incidir en la organización de la clase por parte del profesor.

Desde otra perspectiva el contexto puede desencadenar estrategias para el reconocimiento de este mismo contexto por parte de los estudiantes, el caso 1 lo plantea de la siguiente manera: "el contexto como escenario para el aprendizaje", donde según el caso 3 las estrategias deben favorecer "el interés de los estudiantes por aprender, y reconocer las problemáticas que los rodean" ya que "al abordar el contexto en la clase de biología, los estudiantes enriquecen o amplían su visión sobre el mismo", bajo esta posición el contexto se puede entender mejor como el entorno al que profesor y estudiantes pretender darle un nuevo significado en la clase de biología, al salir del aula para su reconocimiento. Ya algunos autores han propuesto la exploración del entorno vinculando diferentes estrategias o enfoques promoviendo mejores aprendizajes en los estudiantes, Castro (2005) propuso que la investigación del entorno natural como estrategia de enseñanza aprendizaje de las ciencias naturales permite que los estudiantes den una mirada diferente a su contexto y a la vez desarrollen aprendizajes de diferentes tipos, por otro lado autores como González (2010) ya se han referido a la importancia de "saltar la valla de la escuela" para acercarse a conceptos como los de la biodiversidad, no solo desde un punto de vista del entorno natural o biológico, sino desde dimensiones como la social, el consumo en relación con la disminución de la biodiversidad, la publicidad y las nociones que manejan los medios de la 
Edición Extra-Ordinaria. ISSN 2027-1034 P.p $867-876$

Memorias del VII Encuentro Nacional de Experiencias en la Enseñanza de la

Biología y la Educación Ambiental y II Congreso Nacional de Investigación en la Enseñanza de la Biología

diversidad biológica como elementos del contexto para abordar con los estudiantes. Frente a esta mirada los profesores resaltan actividades como "el reconocimiento de las plantas del colegio, debate sobre los problemas de la basura de la ciudad, desmentir una noticia publicada el día anterior, uso de espacios del barrio o de instituciones cercanas para desencadenar procesos de investigación escolar (caso 1)" o que "la salida al patio del colegio, un lugar conocido para ellos puede favorecer que los estudiantes construyan el concepto de especie a partir de lo que observan, gracias a esto los estudiantes se dan cuenta de que existe en mayor número la cantidad de especies exóticas que nativas en el patio (caso 3)".

Las estrategias para la transformación y acción sobre el contexto son sin duda más exigentes para la labor de cualquier profesor, se puede asegurar que no aparecen como una simple idea, sino que se corresponden a las intenciones educativas del docente y su discurso pedagógico, es decir, el docente debe reconocer la educación como un proceso que favorece el cambio y no como el medio para perpetuar el modelo ya existente, donde seguramente se encontrará con muchos obstáculos a diferentes escalas donde su compromiso con su labor será fundamental. En este sentido el caso 2 propone para la enseñanza de la biología que el estudiante al "empoderarse del conocimiento y así mismo convertirse en un estudiante critico, reflexivo, capaz de promover soluciones a su realidad es decir a su contexto, pues adquiere argumentos para apoyar los procesos donde él se encuentra inmerso, hasta el punto de generar cambio a su alrededor incluyendo la vinculación de su familia y comunidad, es decir, la enseñanza y el aprendizaje de la biología no tienen como único fin formar ciudadanos con capacidad de interpretar situaciones de cambio biológico (Jiménez, 2003), sino que hay un reto por encima que corresponde al hacer visibles (así sea en una medida muy pequeña) los cambios en el contexto, por lo menos en cuanto a aspectos donde el profesor, estudiantes, escuela y comunidad cercana puedan influir, ya que no se debe desconocer que la acción o solución sobre la mayoría de problemáticas actuales no dependen únicamente de la escuela. Algunas experiencias que los profesores comparten son que "el concepto trasciende pues se propone que en el colegio se hagan campañas de cuidado y conservación algo que para los estudiantes no era relevante y le daban poca importancia (caso 2)", "la elaboración de comederos para aves con el fin de recuperar la avifauna del barrio, elaboración de planes para el manejo de basuras en cada una de las casas, agricultura urbana, uso de plantas medicinales (caso 1)", "en base a ello aportaron la solución a esta problemática, que fue empezar a consultar las especies de plantas nativas que se podrían empezar a sembrar en la huerta del colegio (caso 3)" o finalmente en palabras del caso 4 se "debe ser un motivador para transformar la realidad social de los estudiantes y para que estos se puedan superar".

\section{Conclusiones}


Memorias del VII Encuentro Nacional de Experiencias en la Enseñanza de la Biología y la Educación Ambiental y II Congreso Nacional de Investigación en la Enseñanza de La Biología

Para los docentes participantes de esta investigación, el conocimiento del contexto se constituye desde diferentes significados y perspectivas, donde la propia experiencia, formación, historia de vida y personalidad intervienen en la construcción de representaciones sobre este término. Con algunas diferencias, según los docentes el contexto que más influye en los procesos de enseñanza y de aprendizaje de la biología es el contexto situacional, manteniendo una relación bidireccional con estos procesos, es decir, la enseñanza y aprendizaje de la biología también pueden transformar el contexto.

El conocimiento del contexto se encuentra relacionado de diferentes maneras con el conocimiento de las orientaciones, estrategias y de los estudiantes. En menor medida para estos docentes el contexto encuentra relación con el conocimiento del currículo, la evaluación y otros. Respecto a la relación con las orientaciones se observa como la selección de elementos vinculados al diseño curricular, en especial los contenidos, deben estar asociados no solocon una visión adecuada de la biología, sino también con problemas o situaciones reales del contexto. En lo referente a las estrategias, la vinculación del contexto a este conocimiento favorece la constitución de tres tipos de estrategias: estrategias adaptadas a las características de los estudiantes (por parte del profesor), estrategias de reconocimiento y aprendizaje del contexto, y estrategias para la transformación y acción sobre el contexto (estas dos últimas desarrolladas por los estudiantes).

\section{Bibliografía}

Arnal, J. \& Latorre, A. (1992). Naturaleza de la Investigación Educación, Investigación Educativa: Fundamentos Y Metodologías, Barcelona: Labor, pp. $24-49$.

Bermúdez G. M. A. y De Longhi A. L. (2012), El conocimiento didáctico de contenidos biológicos de Ecología. pp. 19-35. En Flores R. C.(coordinador), Experiencias Latinoamericanas en educación ambiental, $\mathrm{N}$ 35, CECyTECAEIP, Monterrey, México.

Cañal, P. (2004), La enseñanza de la Biología ¿Cuál es la situación actual y qué hacer para mejorarla? Revista Alambique didácticade las ciencias experimentales, (41), 274.

Castro, J. (2005). La investigación del entorno natural: Una estrategia didáctica para la enseñanza-aprendizaje de las ciencias naturales. Universidad Pedagógica Nacional, p.135.ç

Castro, J. Valbuena, E. (2007). ¿Qué biología enseñar y cómo hacerlo? Hacia una resignificación de la biología escolar. Revista TEA, n. 22, pp. 126-145. 
Memorias del VII Encuentro Nacional de Experiencias en la Enseñanza de la Biologia y la Educación Ambiental y II Congreso Nacional de Investigación en la Enseñanza de la Biología

González, M. (2010). Saltar la valla de la escuela para asomarse a la biodiversidad. Papel de la biodiversidad. Centro de investigación para la paz (CIP-ecosocial). Madrid. 22-28.

Jiménez, M. (2003). La enseñanza y el aprendizaje de la biología. En, Enseñar ciencias. Barcelona: Graó, 2003. p. 121-14.

Kind, V. (2009). El Conocimiento Didáctico en la Educación de la Ciencia: Las Perspectivas y el Potencial para el Progreso, 45 (2), Inglaterra, pp. 169 - 204 DOI:10.1080/030576270903142285.

Magnusson, S., Krajcik, J., \& Borko, H., (2002). Nature, Sources and Development of Pedagogical Content Knowledge for Teaching Science Contemporary Trends and Issues in Science Education, 6 (2), 95-132, DOI: 10.1007/0-306-47217-1_4

Shulman, L. (2005). Conocimiento y Enseñanza: Fundamentos de la Nueva Reforma. Revista de Curriculum y Formación del Profesorado. Estados Unidos, 9(2), p 30.

Valbuena, E. (2007). El conocimiento didáctico del contenido biológico: estudio de las concepciones disciplinares y didácticas de futuros docentes de la universidad pedagógica nacional (Colombia). (Tesis doctoral). Universidad Complutense de Madrid. 